\title{
A educação intergeracional no quadro da educação ao longo da vida e do
}

\author{
envelhecimento ativo
}

\section{Intergenerational education within the framework of lifelong education and active ageing}

\author{
Susana Villas-Boas*, Albertina Lima de Oliveira**, Natália Ramos***, Inmaculada Montero**** \\ * Universidade de Coimbra, ** Universidade de Coimbra, *** Universidade Aberta de Lisboa, **** Universidade de
} Granada

\begin{abstract}
Resumo
No presente trabalho apresenta-se a educação intergeracional, concebida como um processo pedagógico que coloca pessoas de diferentes gerações a executarem atividades e tarefas que respondem às suas necessidades $\mathrm{e}$ interesses, numa dinâmica de participação, cooperação, interação, intercâmbio e de diálogo intergeracional, orientada sob os princípios da relação igualitária, de tolerância e respeito mútuo. A educação intergeracional tem como finalidade principal facilitar e garantir que as pessoas de diferentes gerações aprendam, desenvolvam e compartilhem conhecimentos, competências, habilidades, atitudes e valores e se transformem na relação umas com as outras. Neste sentido, defende-se que se trata de uma abordagem coerente e valiosa, de cariz emancipatório, para o desenvolvimento da educação e aprendizagem ao longo da vida e para a promoção do envelhecimento ativo.

Palavras chave: Educação intergeracional, Educação ao longo da vida, Envelhecimento ativo.
\end{abstract}

\begin{abstract}
This work presents intergenerational education framed as an educational process that puts together people of different generations performing tasks and activities that respond to their needs and interests in a dynamic of participation, cooperation, interaction, exchange and dialogue between generations, oriented under principles of egalitarian relationship, tolerance and mutual respect. Intergenerational education has as main purpose to facilitate and ensure that people of different generations learn, develop and share knowledge, skills, abilities, attitudes and values and become transformed in relation with each other. In this sense, argues that it is a coherent and valuable approach, emancipating oriented, aiming to the development of education and lifelong learning and the promotion of active ageing.

Keywords: Intergenerational education, Lifelong
\end{abstract} education, Active aging.

\section{Introdução}

Pela primeira vez na história da humanidade assistimos a um envelhecimento demográfico sem precedentes, sendo a grande longevidade atual de muitos cidadãos considerada um dos sucessos mais surpreendentes da humanidade. Para além de cada vez mais pessoas viverem mais anos, também vivem com menos incapacidades e limitações funcionais do que as gerações precedentes. Segundo as projeções estatísticas, o envelhecimento demográfico nos países desenvolvidos e em desenvolvimento acentuar-se-á ainda mais nas próximas décadas (INE, 2009; Eurostat, 2013), em consequência do declínio da natalidade, do aumento da esperança de vida, da redução da mortalidade e dos avanços terapêuticos e dos cuidados de saúde. $O$ envelhecimento populacional a que assistimos é um fenómeno universal e irreversível (ONU, 2013). No que diz respeito ao envelhecimento da população portuguesa, em $2013,20 \%$ da mesma tinha 65 ou mais anos (e 5\%, 80 ou mais), prevendo-se que em 2050 estes valores subam para $35 \%$ e $16 \%$ respetivamente (European Commission, 2014). Das estatísticas da PORDATA (www.pordata.pt), verifica-se que Portugal em 2015 era o $5^{\circ}$ país mais envelhecido da Europa (com um índice de envelhecimento de 143,9) e que a esperança de vida à nascença, para os homens era de 78,1 e de 84,3 para as mulheres - superior mas muito semelhante à média europeia (28 Estados membros) que para a mesma data registava 77,9 para os homens e 83,3 para as mulheres.

Nestes termos, podemos dizer que vivemos hoje uma autêntica revolução gerontológica que desafia a reorganização de todos os sistemas a uma mudança profunda entre os quais destacamos o educativo. Neste novo contexto, a educação já não pode seguir, apenas, o caminho das formas tradicionais de ensino, tem de se ajustar às mudanças e às novas exigências. A educação intergeracional surge então como um caminho de grande viabilidade, razão pela qual concordamos com Carmem Canabillas (2011), quando considera que a aprendizagem ao longo da vida, a importância da cultura e a transmissão de valores, o valor da participação social, o potencial da sociedade do conhecimento e da informação, a diversidade e a versatilidade dos lugares em que o conhecimento é 
transmitido, são oportunidades atuais que suportam o desenvolvimento da intervenção social que dará origem a novos modelos de ação educativa, tal como o da educação intergeracional.

\section{Educação intergeracional}

O termo intergeracional é de si problemático, mas quando combinado com o de educação entramos um "mar de abundantes significados" (Watts, 2017, p. 40). A educação intergeracional é um conceito complexo que se encontra em evolução e que necessita de mais discussão, de estudos empíricos e de fundamentação teórica. Todavia, no nosso estudo sobre a educação intergeracional ${ }^{1}$, sistematizamos e apresentamos a seguinte definição:

A educação intergeracional é um processo pedagógico que coloca pessoas de diferentes gerações a executarem atividades e tarefas que respondem às suas necessidades e interesses, numa dinâmica de participação, cooperação, interação, intercâmbio e de diálogo intergeracional desenvolvido numa relação igualitária, de tolerância e respeito mútuo. Tem como principais finalidades facilitar e garantir que as pessoas de diferentes gerações aprendam, desenvolvam e compartilhem conhecimentos, competências, habilidades, atitudes e valores e se transformem na relação umas com as outras [bem como as suas comunidades]. (VillasBoas, Oliveira, Ramos, \& Montero, 2016, p.133)

Muito resumidamente, este tipo de educação (que passaremos a designar por EI) baseia-se nos seguintes princípios: conecta gerações e promove relações intergeracionais através da transmissão de conhecimento entre as gerações, seja das mais novas para com as mais velhas ou vice-versa; do intercâmbio educacional entre as diferentes gerações resulta o enriquecimento mútuo e o florescimento de comunidades, lugares e locais e, em última instância, das nossas sociedades; é uma educação que envolve as modalidades formal, não-formal e informal, e que se desenvolve em variados contextos, tais como na família, comunidades, educação de adultos, no trabalho, em escolas, universidades, centros de dia, associações e até em espaços livres; fundamenta-se na perspetiva da educação ao longo da vida, o que significa desde o berço até à morte, contemplando os primeiros anos de educação, a vida profissional e todas as outras esferas da educação de adultos, que agora se prolonga para a idade adulta avançada, visando integrar plenamente as pessoas idosas.

Arquitetada nos princípios que se acabaram de enunciar, a EI contribui para: melhorar as relações intergeracionais; gerar mais respeito mútuo entre as gerações; aumentar a reciprocidade entre as gerações; aumentar a cooperação entre as diferentes gerações; aumentar a participação e implicação dos indivíduos na

1 Tese de doutoramento intitulada "Educação Intergeracional como Estratégia de Promoção do Envelhecimento Ativo" comunidade e sociedade; diminuir o declínio cognitivo que normativamente acontece com o processo de envelhecimento; aumentar o bem-estar e a qualidade de vida dos indivíduos; aumentar a coesão social; estabelecer redes sociais e redes de apoio social que favoreçam a integração social; promover a educação para a saúde, a educação cívica, a educação familiar, a educação ambiental, entre outros aspetos não tão frequentemente encontrados na literatura.

A educação atual, quer a educação de crianças e jovens quer a educação de adultos, está com grandes dificuldades em conseguir responder às demandas educativas e sociais (Seixas, Oliveira, Alcoforado, \& Reis, 2016), pelo que a EI se apresenta com um grande potencial - no caso das crianças e jovens, pode desempenhar um importante papel complementar à educação formal e, no caso da educação de adultos, ser a resposta humanista, em falta, e ser a oportunidade para a educação dos adultos idosos, ainda tão negligenciada. No próximo ponto debruçamo-nos sobre a EI como parte integrante da educação ao longo da vida.

\section{Educação intergeracional no quadro da educação ao longo da vida}

Os conceitos de educação ao longo da vida e da aprendizagem ao longo da vida são muitas vezes usados como sinónimos (Oliveira, 2015; Gijón, 2016), no entanto, o primeiro foi dominante na literatura educacional até ao final da década de oitenta do século $\mathrm{XX}$, enquadrado numa matriz humanista e solidária, e o segundo tornou-se dominante na literatura a partir dos anos 90 do século passado, até aos nossos dias, passando a estar imbuído de uma ideologia neoliberal, economicista e individualista (Oliveira, 2015). As críticas a este modelo, assente na teoria do capital humano, têm sido numerosas, contestando firmemente: uma globalização educacional, que favorece os países desenvolvidos em detrimento dos países menos desenvolvidos; a imposição do sistema capitalista, favorecendo as classes sociais elitistas, em detrimento da maioria a população; a hegemonia de um modelo que trata o conhecimento e as pessoas como matéria-prima; a persistente desigualdade de acesso à educação para todos, ao longo da vida; que se formem cidadãos com pouca consciência crítica, que aceitam a estrutura económica e social atual, mais do que cidadãos orientados para o progresso económico, político e social, etc. (Cortella, 2000; Lima, 2007, 2016; Gijón, 2016).

Em uníssono com muitos autores (e.g., Lima, 2007; Gijón, 2016), consideramos que é fundamental que se retome e atualize, criticamente, a expressão educação ao longo da vida, considerada em toda a sua amplitude, comprometida com a emergência de sujeitos democráticos, cidadãos livres e autónomos, capazes de fazer uma leitura crítica do mundo e de usar a palavra e a ação coerente com vista à sua transformação. Uma educação de efetiva igualdade de oportunidades "que prepare as pessoas para enfrentarem com sucesso as tarefas e responsabilidades da sua existência" (Lengrand, 1981, cit. Lima, 2007, p. 33). A nosso ver, este retorno tem de partir da realidade atual, de outra 
forma receamos que a nossa visão não passe de uma utopia e nunca concretizável. Assim, regressar à educação humanista significa reinterpretar os relatórios da UNESCO de Edgar Faure et al. (1972), "Aprender a ser", e de Jacques Delors et al. (1996), "Educação, Um tesouro a descobrir" (UNESCO, 1996), em que a educação e a aprendizagem não são considerados conceitos antagónicos mas sim conceitos com um vínculo indissolúvel. Licínio Lima (2007), afirma que entre o modelo do capital humano radicalmente realista e funcionalista e o modelo humanista crítico e emancipatório existe lugar para modalidades híbridas. No nosso entender, a EI, tal como antes caracterizada, ajusta-se bem a esta proposta.

A EI constitui um quadro para o desenvolvimento de uma abordagem coerente e integrada para a educação ao longo da vida (Ohsako, 2002) e contribui para a realização dos seus objetivos. Seja através das modalidades de educação formal, informal, ou não formal, implementadas como parte dos programas intergeracionais ou como método pedagógico, a EI:

- Promove a cultura de educação ao longo da vida. Reforça o conhecimento, de todas as gerações, de que o ser humano aprende ao longo da vida e em todos os contextos de vida. Este reconhecimento do valor e significado da educação ao longo da vida, por parte dos cidadãos, é um pré-requisito e também uma força motriz para a criação de uma cultura de educação ao longo da vida (Ohsako, 2002).

- Promove a consciência sobre a diversidade das culturas das diferentes gerações. Neste processo identificam-se diferenças culturais entre gerações no que diz respeito às suas atitudes, valores e práticas, como também se identificam semelhanças. Procura-se desenvolver não só a aceitação do outro como ajudar os participantes a integrar-se no mundo, mudar pensamentos, rotinas, tradições e estilos de vida, etc., benéficos para todos, resultante da relação com indivíduos de outras gerações.

- Desenvolve atitudes positivas entre gerações. Ao colocar as diferentes gerações em ações ou atividades que partem dos interesses e necessidades comuns a todas as pessoas participantes e em relações de respeito mútuo e não hierárquicas, criam a oportunidade para que as diferentes gerações se conheçam melhor, se desconstruam estereótipos e preconceitos recíprocos, se aumente a tolerância às diferenças e se desenvolvam atitudes positivas entre as diferentes gerações.

- Fomenta a aprendizagem integrada. Ao integrar o pensamento, o sentimento e a ação, num contexto de suporte e apoio, e facultando a liberdade aos participantes de escolherem os conteúdos e as atividades a realizar, a EI possibilita uma aprendizagem que integra todos os componentes da personalidade, física, intelectual, emocional, ética (Faure et al., 1972, p. 156), estética e espiritual (Delors et al., 1996, p. 245).
- É uma educação multidimensional. A EI, para além de ser colocada em prática através da educação formal, não-formal e informal, também cria um vínculo entre os diferentes tipos de ensino e aprendizagem.

- Aproxima a escola e a comunidade. Os programas educativos intergeracionais levam a escola até à comunidade (por exemplo, com programas service learning) ou a comunidade até à escola (por exemplo, com programas de mentoring).

- Permite explorar os interesses e necessidades nas diferentes fases do percurso escolar, de carreira ou reforma. Todos os indivíduos têm talentos, aos quais nem sempre a educação formal responde. A EI parte dos interesses dos indivíduos e identifica necessidades e potencialidades dos mesmos, transformando-se numa oportunidade para recuperar e reorientar os indivíduos em todas as fases das suas vidas.

- Beneficia indivíduos, comunidades e sociedades. Os programas intergeracionais beneficiam tanto indivíduos como as comunidades.

- A EI permite implementar os quatro pilares em que se apoia a educação ao longo da vida (Bedmar, 2003; Mínguez, 2003; Villas-Boas et al., 2014, 2016):

1) Ensina a viver juntos: num ambiente de cooperação e participação entre todas as pessoas envolvidas, ensina a diversidade, a tolerância, conserva tradições e a identidade coletiva, favorece a solidariedade, evita a violência, etc.

2) Ensina a conhecer: fornece meios para adquirir conhecimentos e para compreender o mundo, informa-se, difundem-se notícias e ideias, transmitem-se sentimentos e costumes, desenvolvem-se capacidades profissionais e comunicacionais, etc.

3) Ensina a fazer: desenvolve as competências individuais, através da aprendizagem ativa, colaborativa e experiencial, do trabalho em equipa, do trabalho voluntário, do confrontar e solucionar conflitos, da comunicação empática, etc.

4) Ensina a ser: neste processo as pessoas em contacto umas com as outras passam a conhecer-se melhor a si mesmas e aos outros, desenvolvem o pensamento crítico e autónomo, a criatividade, a responsabilidade, a arte, a cultura, o sentimento de pertença à nossa humanidade comum, etc.

Recentemente, a UNESCO vinculou a educação ao longo da vida a três eixos de desenvolvimento humano, são eles, a saúde e o bem-estar, o emprego e o mercado de trabalho, e a vida social, comunitária e cívica 
(UNESCO, 2015a), e começa a delinear duas grandes tendências para a educação ao longo da vida, sendo a educação intergeracional (UNESCO, 2015b) e as comunidades de aprendizagem (UNESCO, 2016) nestas últimas a EI é parte integrante.

Assim, temos esperança que, mesmo no contexto atual, em que o modelo do capital humano predomina, a EI, englobando lógicas de educação cívica, educação comunitária e de desenvolvimento local, e sendo orientada segundo uma tradição humanista e crítica, de emancipação e de consciencialização, passe a ser mais valorizada e alvo de um crescente investimento.

\section{Educação intergeracional no quadro do envelhecimento ativo}

O envelhecimento ativo é um conceito político recente, para o qual contribuíram organizações como a Organização para a Cooperação e o Desenvolvimento Económico (OCDE), a Organização Mundial da Saúde (OMS) e a União Europeia (UE). Foi definido como:

o processo de otimização das oportunidades de saúde, participação e segurança, visando melhorar a qualidade de vida à medida que as pessoas envelhecem (OMS, 2002, p.79).

Adotamos a perspetiva de envelhecimento ativo da OMS, esclarecendo e reforçando que:

- Tem como objetivo principal fazer do envelhecimento uma experiência positiva para todas as pessoas, à medida que envelhecem;

- O termo "ativo" refere-se a participação nos assuntos sociais, económicos, culturais, espirituais e civis, e não apenas em ser ativo fisicamente e à participação no trabalho;

- Reconhece que é necessário considerar o ciclo de vida;

- Reconhece que os idosos não são um grupo homogéneo e a diversidade do indivíduo tende a aumentar com a idade;

- Tem uma visão multidimensional do envelhecimento, considera que fatores económicos, sociais, ambientais, físicos, comportamentais (estilos de vida), género e a cultura, são determinantes de um envelhecimento ativo;

- Responsabiliza não só o indivíduo, mas também, o coletivo pelo envelhecimento ativo;

- Advoga que o envelhecimento deve ser acompanhado de oportunidades contínuas que contribuam para expandir a esperança de continuar a viver uma vida saudável e com qualidade.
Para que se entenda melhor a necessidade das sociedades atuais (cada vez mais envelhecidas) e dos indivíduos promoverem o envelhecimento ativo, elaborámos o gráfico 1, no qual comparamos os dados relativos à vida saudável depois dos 65 anos de idade de homens e mulheres de 3 países europeus, a saber: Portugal (com um índice de envelhecimento de 143,9); Itália, que é o país mais envelhecido da Europa (cujo índice de envelhecimento é 159,5); e o Luxemburgo, que é o país menos envelhecido da Europa (com um índice de envelhecimento de 85,8 ).

\section{Gráfico 1:}

Anos de vida sã depois dos 65 anos, de homens $e$ mulheres, de 3 países europeus

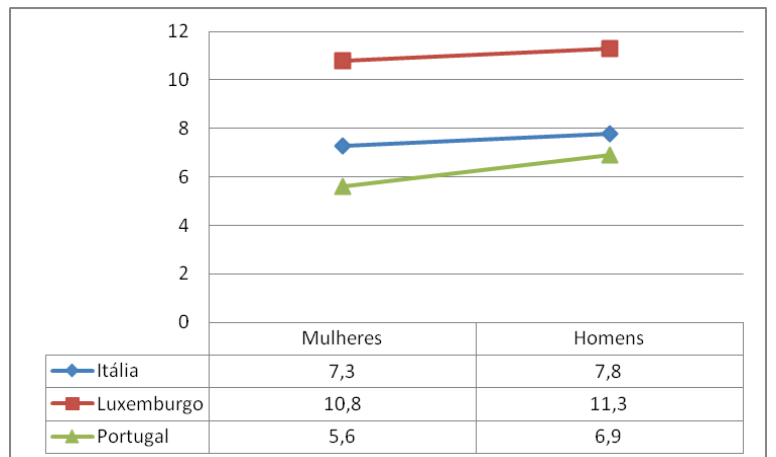

Fonte: PORDATA (www.pordata.pt), acedido a 15 de junho de 2017

Tendo presente, por um lado, que o grande objetivo do envelhecimento ativo é reduzir o número de anos que os indivíduos vivem doentes ou incapacitados antes de morrer, que, como se verifica, acontece em todos os países e, por outro lado, que a esperança média de vida ao nascer dos homens (78,1 anos) e das mulheres (84,3 anos) portuguesas é mais elevada do que a esperança média de vida ao nascer das mulheres (83,3 anos) e dos homens (77,9 anos) da União Europeia (28 Estados membros), podemos afirmar que tanto os homens como as mulheres portuguesas vivem mais anos com pouca saúde e, indiretamente, com menor qualidade de vida e bem-estar do que os nossos conterrâneos europeus, pelo que promover formas de mudar esta realidade não é só um ideal mas uma obrigação.

A EI pode contribuir da seguinte forma:

- Educar e informar as pessoas para o envelhecimento ativo;

- $\mathrm{Na}$ prevenção do envelhecimento ativo ao longo do ciclo vital;

- Dar oportunidades às pessoas para serem reconhecidas pelos outros e pela sociedade, com atividades sociais que favoreçam a sua contribuição para o desenvolvimento das suas comunidades;

- Ajudar as pessoas a aceitar as mudanças naturais do envelhecimento e a saber integrálas nas suas vidas; 
- Ajudar a encontrar formas de 'ajustamento' às limitações;

- Tomar iniciativas para que se criem redes de apoio social entre as pessoas e relações de amizade;

- Através da comunicação, reflexão e escuta ativa das pessoas, pode ajudar a contribuir para investigar outras formas de envelhecer ativamente e contribuir para o desenvolvimento de políticas que promovam o envelhecimento ativo de todas as pessoas.

A OMS refere os programas intergeracionais como um tipo de atividade social que pode promover o envelhecimento ativo pessoal e coletivo (OMS, 2002) e os benefícios encontrados nas investigações sobre este tipo de programas comprovam, efetivamente, que a EI fomenta o envelhecimento ativo, com as características que atrás enunciámos.

\section{Alguns exemplos dos benefícios dos programas educativos intergeracionais.}

Beneficios para as crianças e jovens:

- Melhoram os resultados académicos (Osborne, Weadick \& Penticuff, 1998; Astin, Vogelgesang, Ikeda \& Yee, 2000; Simons \& Cleary, 2006);

- aumentam o desenvolvimento pessoal (Eyler \& Giles, 1999; Astin et al. 2000; Eyler, Giles, Stenson \& Gray, 2001);

- aumentam o desenvolvimento interpessoal (Eyler \& Gile, 1999; Astin et al., 2000; Eyler et al., 2001; Simons \& Cleary, 2006);

- estão associados a um maior conhecimento sobre os adultos idosos e os problemas que enfrentam (Beling, 2003; Karasik, Maddox \& Wallingford, 2004; Ames \& Diepstra, 2006; Zucchero, 2011).

\section{Beneficios para os adultos idosos:}

- Melhoram a saúde (Granville, 2000; Fried et al., 2004; Hernandez \& Gonzalez, 2008; Young \& Janke, 2013; Teater, 2016);

- reduzem a autoperceção negativa e aumentam a autoestima (Underwood \& Dorfman, 2006; Hernandez \& Gonzalez, 2008; Young \& Janke, 2013);

- aumentam as habilidades sociais e a confiança (Teater, 2016);

- eleva-se o sentido para a vida e o reconhecimento por parte dos outros (Granville,2000);

- melhoram a sua qualidade de vida (Hegman et al., 2002);

- melhoram os resultados nos testes de memória (Fried, Carlson, Freedman, Frick, Glass, Hill \& Seedman, 2004; Holmes, 2009);

- criam amizades (Holmes, 2009).

\section{Benéficos para a comunidade:}

- Enriquecem e reconstroem novas redes sociais e melhoram os comportamentos e as atitudes individuais que influenciam na participação na comunidade (Boström, 2002; Kerka, 2003);

- reduzem estereótipos, melhoram a compreensão mútua e a confiança, e promovem a inclusão social, assim como a assimilação de valores (Granville \& Ellis, 1999; Granville, 2000; Kaplan, 2001; Schwalbach \& Kiernan, 2002; Kerka, 2003);

- aumentam a compreensão intergeracional e intercultural (Granville, 2002; Underwood \& Dorfman, 2006);

- melhoram a vida da população (Granville, 2002).

\section{Conclusão}

Pensamos que o envelhecimento da população é uma oportunidade para impulsionar o retorno da perspetiva humanista da educação ao longo da vida, uma educação que pretende contribuir para a criação de cidadãos ativos, que está mais interessada na conexão e cooperação do que na competitividade, em construir um capital social e não o capital humano e que atribui um papel duplo à educação, o da mudança individual e social. É nestas coordenadas que se inscreve a EI, uma educação que tem o potencial de promover a educação cívica, a educação comunitária e o desenvolvimento local, a educação orientada segundo uma tradição crítica, de emancipação e de consciencialização. Neste trabalho advogou-se e procurou-se fundamentar que a EI é um meio para alcançar os objetivos, quer da educação ao longo da vida quer do envelhecimento ativo, pelo que justifica um sério e crescente investimento, bem como comprometimento, por parte de investigadores, educadores, políticos, organizações e instituições locais.

\section{Referencias}

Bedmar, Matías (2003). La educación intergeracional encierra un tesoro. In Matías Bedmar Moreno \& Inmaculada Montero García (Coords.), La educación intergeneracional: un nuevo ámbito educativo (pp. 6782). Madrid: Dykinson.

Cabanillas, C. (2011) Intergenerational learning as an opportunity to generate new educational models. Journal of Intergenerational Relationships, 9(2), 229231. doi: 10.1080/15350770.2011.568347

Comissão Europeia (2014), The 2015 Ageing Report. Brussels: European Commission. Acedido em junho de 2017. Disponível em: http://ec.europa.eu/economy_finance/publications/eur opean_economy/2015/pdf/ee3_en.pdf

Cortella, M. (2000). A escola e o conhecimento. Fundamentos epistemológicos e políticos. São Paulo: Cortez/Instituto Paulo Freire.

Delors, J. (Eds.) (1996). Learning: The treasure within. Report to UNESCO of the International Commission on Education for the Twenty-first Century (José 
Eufrázio Trad.) São Paulo: UNESCO/Edições ASA/Cortez. (Obra original publicada em ano de publicação)

Eurostat (2013). European social statistics, pocketbooks. Luxembourg: Publications Office of the European. Acedido em junho de 2017. Disponível em: http://ec.europa.eu/eurostat/documents/3930297/5968 986/KS-FP-13-001-EN.PDF/

Faure, E., Herrera, F., Kaddoura, A. Lopes, H., Petrovsky, A., Rahnema, M. \& Ward, F. (1972). Learning to be: The World of education today and tomorrow. Paris: UNESCO.

Gijon, J. (2016), Aprendizaje y Educación de Adultos: evolución, tendencias y organización. Granada: Técnica Avicam.

Instituto Nacional de Estatística (INE). (2009). Projeções de população residente em Portugal 20082060. Lisboa: INE, IP. Acedido em Junho de 2017. Disponível em: http://ine.pt/xportal/xmain?xpid=INE\&xpgid=ine_pub licacoes\&PUBLICACOES

Kaplan, M. (2001). Intergenerational programs in schools: Considerations of form and function. International Review of Education, 48(5), 305-334.

Lengrand, P. (1981). Introdução à educação permanente. Lisboa: Livros Horizonte $\left(1^{\mathrm{a}}\right.$ ed. de 1970).

Lima, L. (2007). Educação ao longo da vida: Entre a mão direita e a mão esquerda de Miró. São Paulo: Cortez Editora.

Lima, L. C. (2016). Revisitação gelpiana da educação permanente: Ambiguidades e erosão política de um conceito. Investigar em Educação, 2(5), 53-71. http://pages.ie.uminho.pt/inved/index.php/ie

Mínguez, Constancio (2003). Contenidos y beneficios de la educación inter-generacional. In Matías Bedmar \& Inmaculada Montero (Coords.). La educación intergeneracional: Un nuevo ámbito educativo (pp. 39-65). Madrid: Dykinson.

Ohsako, T. (2002). The role of inter-generational programs in promoting lifelong learning for all ages. In Mendel-Añonuevo, Carolyn (Ed.). Integrating lifelong learning perspectives (pp. 186-207). Hamburg: UNESCO Institute for Education.

Oliveira, A. L. (2015). A autonomia na aprendizagem e a educação e aprendizagem ao longo da vida: A importância dos fatores sociológicos. Revista Práxis Educacional, 11(20), 165-188. http://periodicos.uesb.br/index.php/praxis/article/view File/5279/5060

ONU (2013). World Population Ageing 2013. New York: UN. Retrieved from http://www.un.org/en/development/desa/population/p ublications/pdf/ageing/WorldPopulationAgeing2013.p df

Organización Mundial de la Salude (2002), Envejecimiento activo: un marco político, Revista Española Geriatría y Gerontología, 37(S2),74-105. Retrieved from http://ccp.ucr.ac.cr/bvp/pdf/vejez/oms_envejecimiento _activo.pdf
Seixas, A. M., Oliveira, A. L., Alcoforado, L., \& Reis, C. (2016). Editorial: A educação e formação de adultos no mundo contemporâneo. Revista Portuguesa de Pedagogia, 50(1), 5-12. doi 10.14195/1647$8614 \quad 50-1$

UNESCO (2015a). Education 2030: Incheon declaration and framework for action towards and equality education and lifelong learning for all. Paris: UNESCO. $\quad$ Retrieved from http://www.uis.unesco.org/Education/Documents/inch eon-framework-for-action-en.pdf

UNESCO (2015b). Learning to FLY: Family oriented literacy education in schools. 2004-2014. Hamburg: Institute for Lifelong Learning. Retrieved from http://uil.unesco.org/literacy-and-basic-

skills/engaging-families/learning-fly-family-orientedliteracy-education-schools

UNESCO (2016). Community-based lifelong learning and adult education: Role of community learning centres as facilitators of lifelong learning. Paris: UNESCO. Retrieved from http://unesdoc.unesco.org/images/0024/002467/24674 2E.pdf

Villas-Boas, S., Oliveira, A., Ramos, N., \& Montero, I. (2016). A educação intergeracional no quadro da educação ao longo da vida - Desafios intergeracionais, sociais e pedagógicos. Revista Investigar em Educação, 5, 118-141

Villas-Boas, S., Oliveira, A. \& Ramos, N. (2014). The Inter-generational learning programs: A new sphere of lifelong education. In Conference: Local Change, Social Actions and Adult Learning: Challenges and Responses (pp. 36-43). Lisboa: Institute of Educacion.

Watts, J. (2017), J. Multi or intergenerational learning? Exploring some meanings. Journal of Intergenerational Relationships, 15(1), 39-51. doi:10.1080/15350770.2017.1260367

\section{Agradecimientos}

Co-financiado pela Fundação para a Ciência e Tecnologia. 